\title{
A genetic algorithm-based optimizing approach for project time-cost trade-off with uncertain measure
}

Hua Ke

Correspondence:

hke@tongji.edu.cn

School of Economics and

Management, Tongji University,

Shanghai 200092, China

\begin{abstract}
Both the trade-off between the project cost and the project completion time and the indeterminacy of the environment are important issues for real-life project managers. In this paper, an uncertain time-cost trade-off problem, where activity cost functions are assumed to be linear and the objective function to be minimized is the project direct cost, is described based on uncertainty theory. Two uncertain time-cost trade-off models are built to satisfy different management requirements. To solve the proposed models, two equivalent crisp mathematical programming models are given, and genetic algorithm is introduced to search for quasi-optimal schedules. For future research, resource constraints or more types of indeterminacy can be included.
\end{abstract}

Keywords: Time-cost trade-off; Uncertainty theory; Uncertain measure; Genetic algorithm

\section{Introduction}

For real-life projects, decision-makers should always consider the trade-offs among the performance goals for project scheduling and control, especially the trade-off between project completion time and project cost. The time-cost trade-off problem (TCTP) takes into account the project time-cost trade-off by crashing or prolonging project activity durations. In 1961, Kelly [1] first did research on the TCTP, which is one branch of the project scheduling problem. In the following years, many researches have been done on the deterministic TCTP [2,3]. For solving the deterministic TCTP, the common analytical methods were linear programming and dynamic programming $[4,5]$. Besides, some heuristic algorithms, such as genetic algorithm [6] and simulated annealing algorithm [7], were also applied. In recent years, other important factors for project scheduling, such as quality [8], have also been applied in research on TCTP.

As is well known, the real world is indeterminate. In real-life projects, the activity durations may be variational due to many external factors, such as the increase of productivity level and the change of weather. In recent years, many authors have considered the nondeterministic factors for describing the real-life project indeterminacy. In 1985, Wollmer [9] discussed a stochastic version of the deterministic linear TCTP. In 2000, Gutjahr et al. [10]

(c) 2014 Ke; licensee Springer. This is an Open Access article distributed under the terms of the Creative Commons Attribution License (http://creativecommons.org/licenses/by/4.0), which permits unrestricted use, distribution, and reproduction in any medium, provided the original work is properly credited. 
designed a modified stochastic branch-and-bound approach and applied it to a specific stochastic discrete TCTP. Aghaie and Mokhtari [11] described an approach based on ant colony optimization method and Monte Carlo simulation technique for project crashing problem with exponentially distributed activity durations. Zahraie and Tavakolan [12] embedded two concepts of time-cost trade-off and resource leveling and allocation in a stochastic multiobjective optimization model, where fuzzy set theory was applied to represent different options for each activity. Ke et al. [13] built two models for stochastic TCTP with the philosophies of chance-constrained programming and dependent-chance programming. Mokhtari et al. [14] developed a hybrid optimization approach based on cutting plane method and Monte Carlo simulation for stochastic TCTP in PERT networks. Ke et al. [15] modeled stochastic project time-cost trade-offs with time-dependent activity durations.

For some projects in indeterminate environment, probability theory is no longer valid for describing activity durations for the lack of statistical data, since human beings usually overweigh unlikely events. For this case, the activity durations may be described by fuzzy variables. The first work on the fuzzy TCTP was done by Leu et al. [16]. Jin et al. [17] gave a genetic algorithm (GA)-based fully fuzzy optimal time-cost tradeoff model, in which all parameters and variables were characterized by fuzzy numbers and an example in ship building scheduling was demonstrated. Eshtehardian et al. [18] established a multiobjective fuzzy time-cost model. Ghazanfari et al. [19,20] applied possibilistic goal programming to the TCTP to determine optimal duration for each activity in the form of triangular fuzzy numbers. Ke et al. [21] built three fuzzy programming models for TCTP based on credibility theory. Chen and Tsai [22] constructed membership function of fuzzy minimum total crash cost based on Zadeh's extension principle and transformed the time-cost trade-off problem to a pair of parametric mathematical programs.

When the indeterminacy does not behave either randomly or fuzzily, we need a new tool to deal with it. To describe indeterminacy which is neither randomness nor fuzziness, Liu founded uncertainty theory in 2007 [23] and refined it in 2010 [24]. Uncertainty theory is a branch of axiomatic mathematics for modeling human uncertainty. To the knowledge of the authors, no researchers considered time-cost trade-off problem in uncertain environment, which is not either stochastic or fuzzy. In this paper, we introduce uncertainty theory for modeling the TCTP in indeterminate environment. We propose two uncertain time-cost trade-off models according to some different decision-making criteria. As Huang and Ding [25] showed that using standard path algorithms (e.g., the well-known Dijkstra method) was not able to arrive at solutions for searching critical path of this problem, GA is applied in this paper. Some numerical experiments in which the activity durations are assumed to be uncertain variables with known uncertainty distributions are presented.

The remainder of the paper is organized as follows: Section 'Preliminaries' gives some concepts of uncertainty theory as preliminaries for modeling. Section 'Problem description' describes the TCTP with uncertain activity durations. In Section 'Uncertain models of time-cost trade-off problem', based on some decision-making criteria, two types of uncertain models are presented. The following section gives two numerical experiments to illustrate the proposed models. Finally, Section 'Conclusions' draws some concluding statements. 


\section{Preliminaries}

To better describe and understand uncertain phenomena, Liu [23] proposed uncertainty theory, which has been applied to many fields such as uncertain calculus [26,27], uncertain risk analysis [28], uncertain logic [29], uncertain finance [30], and uncertain differential equation [31]. Based on uncertainty theory, Liu [32] formulated uncertain programming for solving application problems with uncertain factors, which has been applied into some optimization problems, e.g., option pricing [33], facility location [34], inventory problem [35], transportation problem [36], uncertain graph [37], and portfolio selection [38].

In this section, we introduce some basic concepts which will be helpful for establishing some uncertain models for the TCTP. Let $\Gamma$ be a nonempty set, and $\mathcal{L}$ a $\sigma$-algebra over $\Gamma$. Each element $\Lambda$ in $\mathcal{L}$ is called an event.

Definition 1. (Liu [23]) The set function $\mathcal{M}$ is called an uncertain measure if it satisfies:

Axiom 1. $\mathcal{M}\{\Gamma\}=1$ for the universal set $\Gamma$.

Axiom 2. $\mathcal{M}\{\Lambda\}+\mathcal{M}\left\{\Lambda^{c}\right\}=1$ for any event $\Lambda$.

Axiom 3. For every countable sequence of events $\Lambda_{1}, \Lambda_{2}, \ldots$, we have

$$
\mathcal{M}\left\{\bigcup_{i=1}^{\infty} \Lambda_{i}\right\} \leq \sum_{i=1}^{\infty} \mathcal{M}\left\{\Lambda_{i}\right\} .
$$

Besides, the product uncertain measure on the product $\sigma$-algebra $€$ was defined by Liu [26] as follows:

Axiom 4. Let $\left(\Gamma_{k}, \mathrm{I}_{k}, \mathcal{M}_{k}\right)$ be uncertainty spaces for $k=1,2, \ldots$. The product uncertain measure $\mathcal{M}$ is an uncertain measure satisfying

$$
\mathcal{M}\left\{\prod_{k=1}^{\infty} \Lambda_{k}\right\}=\bigwedge_{k=1}^{\infty} \mathcal{M}_{k}\left\{\Lambda_{k}\right\}
$$

where $\Lambda_{k}$ are arbitrarily chosen events from $\iota_{k}$ for $k=1,2, \ldots$, respectively.

Based on the definition of uncertain measure, we can give the concept of an uncertain variable.

Definition 2. (Liu [23]) An uncertain variable is a measurable function $\xi$ from an uncertainty space $(\Gamma, \mathcal{L}, \mathcal{M})$ to the set of real numbers, i.e., for any Borel set $B$ of real numbers, the set

$$
\{\xi \in B\}=\{\gamma \in \Gamma \mid \xi(\gamma) \in B\}
$$

is an event.

With the concept of uncertain variable, we can define the uncertainty distribution of an uncertain variable.

Definition 3. (Liu [23]) The uncertainty distribution $\Phi$ of an uncertain variable $\xi$ is defined by

$$
\Phi(x)=\mathcal{M}\{\xi \leq x\}
$$

for any real number $x$. 
Liu [24] also defined the inverse function $\Phi^{-1}$ as the inverse uncertainty distribution of uncertain variable $\xi$. With inverse uncertainty distribution, Liu [24] gave the operational law of uncertain variables as follows.

Theorem 1. (Liu [24]) Let $\xi_{1}, \xi_{2}, \ldots, \xi_{n}$ be independent uncertain variables with regular uncertainty distributions $\Phi_{1}, \Phi_{2}, \ldots, \Phi_{n}$, respectively. If the function $f\left(x_{1}, x_{2}, \ldots, x_{n}\right)$ is strictly increasing with respect to $x_{1}, x_{2}, \ldots, x_{m}$ and strictly decreasing with respect to $x_{m+1}, x_{m+2}, \ldots, x_{n}$, then

$$
\xi=f\left(\xi_{1}, \xi_{2}, \ldots, \xi_{n}\right)
$$

is an uncertain variable with inverse uncertainty distribution

$$
\Phi^{-1}(\alpha)=f\left(\Phi_{1}^{-1}(\alpha), \ldots, \Phi_{m}^{-1}(\alpha), \Phi_{m+1}^{-1}(1-\alpha), \ldots, \Phi_{n}^{-1}(1-\alpha)\right) .
$$

For giving out some decision-making criteria for managers, we introduce the following definition:

Definition 4. (Liu [23]) Let $\xi$ be an uncertain variable. The expected value of $\xi$ is defined by

$$
E[\xi]=\int_{0}^{+\infty} \mathcal{M}\{\xi \geq r\} \mathrm{d} r-\int_{-\infty}^{0} \mathcal{M}\{\xi \leq r\} \mathrm{d} r
$$

provided that at least one of the above two integrals is finite.

Theorem 2. (Liu [24]) Let $\xi$ be an uncertain variable with regular uncertainty distribution $\Phi$. If the expected value exists, then

$$
E[\xi]=\int_{0}^{1} \Phi^{-1}(\alpha) \mathrm{d} \alpha .
$$

For instance, let $\xi \sim \mathcal{L}(a, b)$ be a linear uncertain variable. Then its inverse uncertainty distribution is $\Phi^{-1}(\alpha)=(1-\alpha) a+\alpha b$, and its expected value is

$$
E[\xi]=\int_{0}^{1}((1-\alpha) a+\alpha b) \mathrm{d} \alpha=\frac{a+b}{2} .
$$

\section{Problem description}

With the project progress, project managers always need to make a trade-off between the cost and the completion time. Sometimes managers may make a decision in order to finish the project sooner with project cost augmentation by accelerating the project schedule, which is also named as project crashing in project management. In other cases, motivated by reducing project cost, managers may be conscripted to sacrifice by prolonging the project completion time. Therefore, it is naturally desirable for managers to find a schedule to complete a project with the balance of the cost and the completion time.

A project can be represented by an activity-on-arc network $G=(V, A)$, where $V=$ $\{1,2, \ldots, n\}$ is the set of nodes representing the milestones and $A$ is the set of arcs representing the activities, shown as Figure 1. In the network, nodes 1 and $n$ represent the start and the end of the project, respectively. In this paper, the normal activity durations are assumed to be uncertain variables. Note that the normal duration of activity $(i, j)$ denoted 


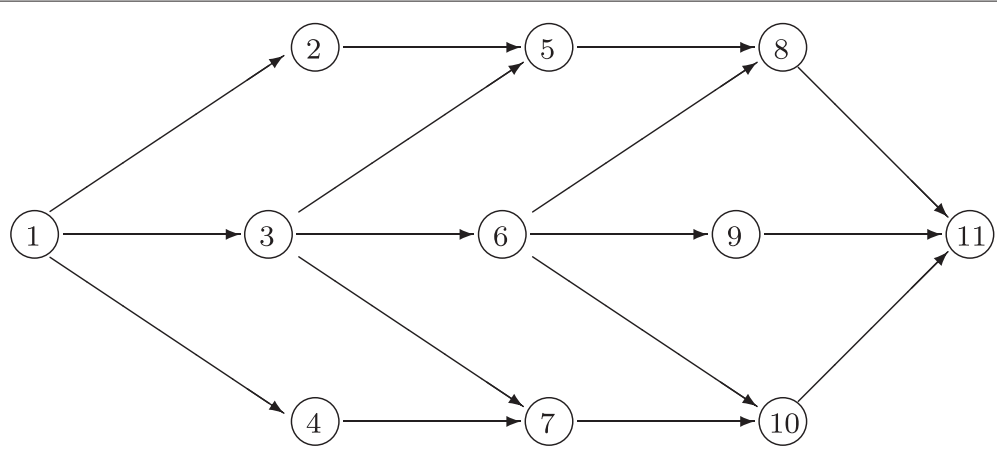

Figure 1 A project. Nodes 1 and $n$ represent the start and the end of the project, respectively.

as $\xi_{i j}$ represents the duration without the influence of the decision made by the manager, and the uncertainty of $\xi_{i j}$ is derived from the uncertain project environment. Correspondingly, the normal cost per time unit of activity $(i, j)$ is denoted by $c_{i j}$. The decision variable $x_{i j}$ indicates the duration change of activity $(i, j)$ controlled by the manager, such as determining the number change of workers and changing the instruments. The variable $x_{i j}$, supposed to be an integer for the sake of simplicity, is bounded by some interval $\left[l_{i j}, u_{i j}\right]$ owing to practical conditions, where $l_{i j}$ and $u_{i j}$ are also assumed to be integers. For each activity $(i, j)$, there also exists another associated cost $d_{i j}$, regarded as the additional cost of per unit change of $x_{i j}$. For simplicity, $c_{i j}$ and $d_{i j}$ are both assumed to be constants.

Then, for the trade-off between the completion time and the cost, the goal is to decide the optimal vector $\boldsymbol{x}=\left\{x_{i j}:(i, j) \in A\right\}$ to meet different scheduling requirements.

The uncertain normal activity durations can be concisely written as $\xi=\left\{\xi_{i j}:(i, j) \in A\right\}$. The starting time of activity $(i, j)$ is denoted by $T_{i j}(\boldsymbol{x}, \boldsymbol{\xi})$, and the starting time of activity $(1, j) \in A$ is defined as $T_{1 j}(\boldsymbol{x}, \boldsymbol{\xi})=0$, which means that the starting time of the total project is assumed to be 0 . To simplify the problem, we assume that each activity can be processed only if all the foregoing activities are finished and should be processed without interruption, and no lead or lag times are possible. With the assumptions, the starting time of activity $(i, j), i=2,3, \ldots, n-1$ can be calculated by

$$
T_{i j}(\boldsymbol{x}, \boldsymbol{\xi})=\max _{(k, i) \in A}\left\{T_{k i}(\boldsymbol{x}, \boldsymbol{\xi})+\xi_{k i}+x_{k i}\right\} .
$$

Suppose that $T_{i j}(\boldsymbol{x}, \boldsymbol{\xi})$ has an inverse uncertainty distribution $\Psi_{i j}^{-1}(\boldsymbol{x}, \alpha)$. Then

$$
\Psi_{i j}^{-1}(\boldsymbol{x}, \alpha)=\max _{(k, i) \in A}\left\{\Psi_{k i}^{-1}(\boldsymbol{x}, \alpha)+\Phi_{k i}^{-1}(\alpha)+x_{k i}\right\},
$$

and the project completion time can be written by

$$
T(\boldsymbol{x}, \boldsymbol{\xi})=\max _{(k, n) \in A}\left\{T_{k n}(\boldsymbol{x}, \boldsymbol{\xi})+\xi_{k n}+x_{k n}\right\}
$$

whose inverse uncertainty distribution is

$$
\Psi^{-1}(\boldsymbol{x}, \alpha)=\max _{(k, n) \in A}\left\{\Psi_{k n}^{-1}(\boldsymbol{x}, \alpha)+\Phi_{k n}^{-1}(\alpha)+x_{k n}\right\} .
$$

The project cost, composed of the normal cost and the additional cost, can be simply computed by

$$
C(\boldsymbol{x}, \boldsymbol{\xi})=\sum_{(i, j) \in A}\left(c_{i j} \xi_{i j}-d_{i j} x_{i j}\right)
$$


whose inverse uncertainty distribution is

$$
\Upsilon^{-1}(\boldsymbol{x}, \alpha)=\sum_{(i, j) \in A}\left(c_{i j} \Phi_{i j}^{-1}(\alpha)-d_{i j} x_{i j}\right) .
$$

\section{Uncertain models of time-cost trade-off problem}

In many researches on indeterminate decision systems, optimizing expected objective value is preferably considered to be the choice for decision-making and expected value model is the most employed model. However, for various practical requirements, other alternative decision-making criteria and optimization models are needed. In this paper, except the expected value model, one more optimization model for the uncertain TCTP is presented with the philosophy of dependent-chance programming.

\section{Expected cost minimization model}

As we mentioned above, comparing expected values is the most widely applied decisionmaking criterion in practice. Risk-averse managers usually want to find the optimal decision with minimum expected project cost subject to some project completion time constraint. With this criterion, we can present the following expected cost minimization model for TCTP:

$$
\left\{\begin{array}{l}
\min E[C(\boldsymbol{x}, \boldsymbol{\xi})] \\
\text { subject to } \\
\mathcal{M}\left\{T(\boldsymbol{x}, \boldsymbol{\xi}) \leq T^{0}\right\} \geq \alpha_{0} \\
x_{i j} \in\left[l_{i j}, u_{i j}\right], \forall(i, j) \in A \\
x_{i j}, \forall(i, j) \in A, \text { integers, }
\end{array}\right.
$$

where $T^{0}$ is the due date of the project, $\alpha_{0}$ is a predetermined confidence level, $l_{i j}$ and $u_{i j}$ are integers given in advance, and $T(\boldsymbol{x}, \boldsymbol{\xi})$ and $C(\boldsymbol{x}, \boldsymbol{\xi})$ are defined by (1) and (3), respectively. The above model is equivalent to

$$
\left\{\begin{array}{l}
\min \int_{0}^{1} \Upsilon^{-1}(\boldsymbol{x}, \alpha) \mathrm{d} \alpha \\
\text { subject to } \\
\Psi^{-1}\left(\boldsymbol{x}, \alpha_{0}\right) \leq T^{0} \\
x_{i j} \in\left[l_{i j}, u_{i j}\right], \quad \forall(i, j) \in A \\
x_{i j}, \quad \forall(i, j) \in A, \text { integers, }
\end{array}\right.
$$

where $\Psi^{-1}(\boldsymbol{x}, \alpha)$ and $\Upsilon^{-1}(\boldsymbol{x}, \alpha)$ are determined by (2) and (4), respectively.

\section{Chance maximization model}

In real-life project, the manager may tend to control the project cost within some budget and meanwhile complete the project in time. However, due to the environmental complexity, these performance goals are not always obtained completely. Then it is natural for the project manager to maximize the chance that the project cost does not exceed the given budget under some project completion time constraint, which follows 
the philosophy of dependent-chance programming (DCP) introduced by Liu [39]. Based on the DCP philosophy, we can establish the chance maximization model as follows:

$$
\left\{\begin{array}{l}
\max \mathcal{M}\left\{C(\boldsymbol{x}, \boldsymbol{\xi}) \leq C^{0}\right\} \\
\text { subject to } \\
\mathcal{M}\left\{T(\boldsymbol{x}, \boldsymbol{\xi}) \leq T^{0}\right\} \geq \alpha_{0} \\
x_{i j} \in\left[l_{i j}, u_{i j}\right], \quad \forall(i, j) \in A \\
x_{i j}, \quad \forall(i, j) \in A, \text { integers, }
\end{array}\right.
$$

where $\alpha_{0}$ is a predetermined confidence level, $T^{0}$ is the due date of the project, $C^{0}$ is the budget, $l_{i j}$ and $u_{i j}$ are integers given in advance, and $T(\boldsymbol{x}, \boldsymbol{\xi})$ and $C(\boldsymbol{x}, \boldsymbol{\xi})$ are defined by (1) and (3), respectively. Note that here so-called chance is measured by uncertain measure. This model is equivalent to

$$
\left\{\begin{array}{l}
\max \bar{\beta} \\
\text { subject to } \\
\Upsilon^{-1}(\boldsymbol{x}, \bar{\beta}) \leq C^{0} \\
\Psi^{-1}\left(\boldsymbol{x}, \alpha_{0}\right) \leq T^{0} \\
x_{i j} \in\left[l_{i j}, u_{i j}\right], \quad \forall(i, j) \in A \\
x_{i j}, \quad \forall(i, j) \in A, \quad \text { integers, }
\end{array}\right.
$$

where $\Psi^{-1}(\boldsymbol{x}, \alpha)$ and $\Upsilon^{-1}(\boldsymbol{x}, \beta)$ are determined by (2) and (4), respectively.

\section{Numerical experiments}

By a simple example, Huang and Ding [25] demonstrated that the standard path algorithms (e.g., the well-known Dijkstra method) were not capable of finding the critical path for the random project scheduling problem, which is applicable to uncertain TCTP. Hence, for solving the above two uncertain time-cost trade-off models, GA is introduced to search for the quasi-optimal solutions.

Consider the project network shown in Figure 1. For every activity $(i, j)$, the duration is assumed to be a linear uncertain variable $\mathcal{L}(2 i+3,2 j+2)$. The normal costs and the additional costs of the activities are presented in Table 1 , respectively. We also assume that the decision variable $x_{i j}$ is an integer and limited in the interval $[-4,3]$ for each $(i, j) \in A$.

First, the project manager tends to finish the project in 62 time units with confidence level 0.85 and meanwhile minimizes the project cost in the sense of expected value. With this demand, we can present the following expected cost minimization model:

$$
\left\{\begin{array}{l}
\min E[C(\boldsymbol{x}, \boldsymbol{\xi})] \\
\text { subject to } \\
\mathcal{M}\{T(\boldsymbol{x}, \boldsymbol{\xi}) \leq 62\} \geq 0.85 \\
x_{i j} \in[-4,3], \quad \forall(i, j) \in A \\
x_{i j}, \forall(i, j) \in A, \text { integers. }
\end{array}\right.
$$

This model is equivalent to

$$
\left\{\begin{array}{l}
\min \int_{0}^{1} \Upsilon^{-1}(\boldsymbol{x}, \alpha) \mathrm{d} \alpha \\
\text { subject to } \\
\Psi^{-1}(\boldsymbol{x}, 0.85) \leq 62 \\
x_{i j} \in[-4,3], \quad \forall(i, j) \in A \\
x_{i j}, \quad \forall(i, j) \in A, \quad \text { integers. }
\end{array}\right.
$$


Table 1 Activity costs of project

\begin{tabular}{ccc}
\hline $\begin{array}{c}\text { Activity } \\
(\boldsymbol{i}, \boldsymbol{j})\end{array}$ & $\begin{array}{c}\text { Normal cost } \\
\boldsymbol{c}_{\boldsymbol{i j}}\end{array}$ & $\begin{array}{c}\text { Additional cost } \\
\boldsymbol{d}_{\boldsymbol{i j}}\end{array}$ \\
\hline$(1,2)$ & 170 & 200 \\
$(1,3)$ & 300 & 280 \\
$(1,4)$ & 65 & 70 \\
$(2,5)$ & 270 & 300 \\
$(3,5)$ & 135 & 150 \\
$(3,6)$ & 75 & 90 \\
$(3,7)$ & 150 & 100 \\
$(4,7)$ & 600 & 400 \\
$(5,8)$ & 85 & 100 \\
$(6,8)$ & 300 & 400 \\
$(6,9)$ & 95 & 90 \\
$(6,10)$ & 130 & 140 \\
$(7,10)$ & 200 & 240 \\
$(8,11)$ & 90 & 120 \\
$(9,11)$ & 200 & 180 \\
$(10,11)$ & 320 & 380 \\
\hline
\end{tabular}

For the above model, we can easily employ GA to search for the quasi-optimal solution. We set the parameters in GA as the population size of one generation pop_size $=50$, the probability of mutation $P_{\mathrm{m}}=0.5$, and the probability of crossover $P_{\mathrm{c}}=0.7$. After a run of 8,000 generations, we obtain the quasi-optimal solution $x^{*}=$ $(3,1,-4,3,3,-2,0,2,0,3,1,2,-2,0,0,-2), E\left[C\left(x^{*}, \xi\right)\right]=43,482.5$ and $\mathcal{M}\left\{T\left(x^{*}, \xi\right) \leq\right.$ $62\}=0.857$.

The other considered model is the chance maximization shown as follows:

$$
\left\{\begin{aligned}
& \max \mathcal{M}\{C(\boldsymbol{x}, \boldsymbol{\xi}) \leq 46,700\} \\
& \text { subject to } \\
& \mathcal{M}\{T(\boldsymbol{x}, \boldsymbol{\xi}) \leq 65\} \geq 0.85 \\
& x_{i j} \in[-4,3], \quad \forall(i, j) \in A \\
& x_{i j}, \quad \forall(i, j) \in A, \text { integers. }
\end{aligned}\right.
$$

This model is equivalent to

$$
\left\{\begin{array}{l}
\max \bar{\beta} \\
\text { subject to } \\
\Upsilon^{-1}(\boldsymbol{x}, \bar{\beta}) \leq 46,700 \\
\Psi^{-1}(\boldsymbol{x}, 0.85) \leq 65 \\
x_{i j} \in[-4,3], \quad \forall(i, j) \in A \\
x_{i j}, \quad \forall(i, j) \in A, \text { integers. }
\end{array}\right.
$$

After a run of 6,000 generations with pop_size $=50, P_{\mathrm{m}}=0.6$, and $P_{\mathrm{c}}=0.5$, we obtain the quasi-optimal solution $\boldsymbol{x}^{*}=(2,0,-4,3,2,0,1,2,3,3,0,3,0,1,3,-1)$ and $\mathcal{M}\left\{C\left(x^{*}, \xi\right) \leq 46,700\right\}=0.874$.

\section{Conclusions}

For real-life project managers, both the trade-off between the cost and the completion time and the indeterminacy environment are considerable issues. In this paper, an 
uncertain TCTP was formulated with the objective of minimizing the cost with completion time limits based on uncertain measure. With some decision-making criteria, the expected cost minimization model and the chance maximization model were established to satisfy different practical managing requirements. Two equivalent crisp mathematical programming models were also given. To solve the models, GA was introduced.

The TCTP with uncertain activity durations can be regarded as the extension of the fuzzy TCTP. Furthermore, resource constraints or more types of indeterminacies can be included for future research. For real-life application, the models can be applied to many other project optimization problems.

\section{Acknowledgments}

The work was partly supported by the National Natural Science Foundation of China (71371141, 71001080).

Received: 12 February 2014 Accepted: 25 March 2014

Published: 24 April 2014

\section{References}

1. Kelley, JE: Critical path planning and scheduling mathematical basis. Oper. Res. 9, 296-320 (1961)

2. Tareghian, HR, Taheri, SH: On the discrete time, cost and quality trade-off problem. Appl. Math. Comput. 181, 1305-1312 (2006)

3. Weglarz, J, Jozefowska, J, Mika, M, Waligora, G: Project scheduling with finite or infinite number of activity processing modes - a survey. Eur. J. Oper. Res. 208, 177-205 (2011)

4. Heberta, JE, Deckro, RF: Combining contemporary and traditional project management tools to resolve a project scheduling problem. Comput. Oper. Res. 38, 21-32 (2011)

5. Talbot, FB: Resource-constrained project scheduling with time-resource tradeoffs: the non-preemptive case. Manage. Sci. 28, 1197-1210 (1982)

6. Azaron, A, Perkgoz, C, Sakawa, M: A genetic algorithm approach for the time-cost trade-off in PERT networks. Appl. Math. Comput. 168, 1317-1339 (2005)

7. Anagnostopoulos, KP, Kotsikas, L: Experimental evaluation of simulated annealing algorithms for the time-cost trade-off problem. Appl. Math. Comput. 217, 260-270 (2010)

8. Salmasnia, A, Mokhtari, H, Abadi, INK: A robust scheduling of projects with time, cost, and quality considerations. Int. J. Adv. Manuf. Tech. 60, 631-642 (2012)

9. Wollmer, RD: Critical path planning under uncertainty. Math. Program. Stud. 25, 164-171 (1985)

10. Gutjahr, WJ, Strauss, C, Wagner, E: A stochastic branch-and-bound approach to activity crashing in project management. INFORMS J. Comput. 12, 125-135 (2000)

11. Aghaie, A, Mokhtari, $\mathrm{H}$ : Ant colony optimization algorithm for stochastic project crashing problem in PERT networks using MC simulation. Int. J. Adv. Manuf. Tech. 45, 1051-1067 (2009)

12. Zahraie, B, Tavakolan, M: Stochastic time-cost-resource utilization optimization using nondominated sorting genetic algorithm and discrete fuzzy sets. J. Constr. Eng. M. 135, 1162-1171 (2009)

13. $\mathrm{Ke}, \mathrm{H}, \mathrm{Ma}, \mathrm{W}, \mathrm{Ni}, \mathrm{Y}$ : Optimization models and a GA-based algorithm for stochastic time-cost trade-off problem. Appl. Math. Comput. 215, 308-313 (2009)

14. Mokhtari, H, Aghaie, A, Rahimi, J, Mozdgir, A: Project time-cost trade-off scheduling: a hybrid optimization approach Int. J. Adv. Manuf. Tech. 50, 811-822 (2010)

15. Ke, H, Ma, W, Chen, X: Modeling stochastic project time-cost trade-offs with time-dependent activity durations. Appl. Math. Comput. 218, 9462-9469 (2012)

16. Leu, SS, Chen, AT, Yang, CH: A GA-based fuzzy optimal model for construction time-cost trade-off. Int. J. Proj. Manage. 19, 47-58 (2001)

17. Jin, C, Ji, Z, Lin, Y, Zhao, Y, Huang, Z: Research on the fully fuzzy time-cost trade-off based on genetic algorithms. J. Mar. Sci. Appl. 4, 18-23 (2005)

18. Eshtehardian, E, Afshar, A, Abbasnia, R: Time-cost optimization: using GA and fuzzy sets theory for uncertainties in cost. Constr. Manage. Econ. 26, 679-691 (2008)

19. Ghazanfari, M, Shahanaghi, K, Yousefli, A: An application of possibility goal programming to the time-cost trade off problem. J. Uncertain Syst. 2, 22-28 (2008)

20. Ghazanfari, M, Yousefli, A, Ameli, MSJ, Bozorgi-Amiri, A: A new approach to solve time-cost trade-off problem with fuzzy decision variables. Int. J. Adv. Manuf. Tech. 42, 408-414 (2009)

21. Ke, H, Ma, W, Gao, X, Xu, W: New fuzzy models for time-cost trade-off problem. Fuzzy Optim. Decis. Ma. 9, 219-231 (2010)

22. Chen, S, Tsai, M: Time-cost trade-off analysis of project networks in fuzzy environments. Eur. J. Oper. Res. 212 386-397 (2011)

23. Liu, B: Uncertainty Theory. 2nd ed. Springer, Berlin (2007)

24. Liu, B: Uncertainty Theory: a Branch of Mathematics for Modeling Human Uncertainty. Springer, Berlin (2010)

25. Huang, W, Ding, L: Project-scheduling problem with random time-dependent activity duration times. IEEE T. Eng Manage. 58, 377-387 (2011)

26. Liu, B: Some research problems in uncertainty theory. J. Uncertain Syst. 3, 3-10 (2009)

27. Chen, XW, Ralescu, DA: Liu process and uncertain calculus. J. Uncertain. Anal. Appl. 1, Article 3 (2013)

28. Liu, B: Uncertain risk analysis and uncertain reliability analysis. J. Uncertain Syst. 4, 163-170 (2010)

29. Liu, B: Uncertain logic for modeling human language. J. Uncertain Syst. 5, 3-20 (2011) 
30. Liu, B: Toward uncertain finance theory. J. Uncertain. Anal. Appl. 1, Article 1 (2013)

31. Yao, K: Extreme values and integral of solution of uncertain differential equation. J. Uncertain. Anal. Appl. 1, 2 (2013)

32. Liu, B: Theory and Practice of Uncertain Programming. 2nd ed. Springer, Berlin (2009)

33. Chen, XW: American option pricing formula for uncertain financial market. Int. J. Oper. Res. 8, 32-37 (2011)

34. Gao, Y: Uncertain models for single facility location problems on networks. Appl. Math. Model. 36, 2592-2599 (2012)

35. Rong, LX: Two new uncertainty programming models of inventory with uncertain costs. J. Inf. Comput. Sci. 8, 280-288 (2011)

36. Sheng, Y, Yao, K: Fixed charge transportation problem in uncertain environment. Ind. Eng. Manage. Sys. 11, 183-187 (2012)

37. Zhang, B, Peng, J: Euler index in uncertain graph. Appl. Math. Comput. 218, 10279-10288 (2012)

38. Zhu, Y: Uncertain optimal control with application to a portfolio selection model. Cy. Sys. 41, 535-547 (2010)

39. Liu, B: Dependent-chance programming: a class of stochastic programming. Comput. Math. Appl. 34, 89-104 (1997)

doi:10.1186/2195-5468-2-8

Cite this article as: Ke: A genetic algorithm-based optimizing approach for project time-cost trade-off with uncertain measure. Journal of Uncertainty Analysis and Applications 2014 2:8.

\section{Submit your manuscript to a SpringerOpen ${ }^{\odot}$ journal and benefit from:}

- Convenient online submission

- Rigorous peer review

- Immediate publication on acceptance

- Open access: articles freely available online

- High visibility within the field

Retaining the copyright to your article

Submit your next manuscript at $\boldsymbol{\triangleright}$ springeropen.com 